\section{The Trans-zoonotic Virome interface: Measures to balance, control and treat epidemics}

\author{
Vinod Nikhra* \\ MD, Hindu Rao Hospital \& NDMC Medical College, New Delhi, India
}

\section{Abstract}

The global virome: The viruses have a global distribution, phylogenetic diversity and host specificity. They are obligate intracellular parasites with single- or double-stranded DNA or RNA genomes, and afflict bacteria, plants, animals and human population. The viral infection begins when surface proteins bind to receptor proteins on the host cell surface, followed by internalisation, replication and lysis. Further, trans-species interactions of viruses with bacteria, small eukaryotes and host are associated with various zoonotic viral diseases and disease progression.

Virome interface and transmission: The cross-species transmission from their natural reservoir, usually mammalian or avian, hosts to infect human-being is a rare probability, but occurs leading to the zoonotic human viral infection. The factors like increased human settlements and encroachments, expanded travel and trade networks, altered wildlife and livestock practices, modernised and mass-farming practices, compromised ecosystems and habitat destruction, and global climate change have impact on the interactions between virome and its hosts and other species and act as drivers of trans-species viral spill-over and human transmission.

Zoonotic viral diseases and epidemics: The zoonotic viruses have caused various deadly pandemics in human history. They can be further characterized as either newly emerging or re-emerging infectious diseases, caused by pathogens that historically have infected the same host species, but continue to appear in new locations or in drug-resistant forms, or reappear after apparent control or elimination. The prevalence of zoonoses underlines importance of the animal-human-ecosystem interface in disease transmission. The present COVID-19 infection has certain distinct features which suppress the host immune response and promote the disease potential.

Treatment for epidemics like covid-19: It appears that certain nutraceuticals may provide relief in clinical symptoms to patients infected with encapsulated RNA viruses such as influenza and coronavirus. These nutraceuticals appear to reduce the inflammation in the lungs and help to boost type 1 interferon response to these viral infections. The human intestinal microbiota acting in tandem with the host's defence and immune system, is vital for homeostasis and preservation of health. The integrity and balanced activity of the gut microbes is responsible for the protection from disease states including viral infections. Certain probiotics may help in improving the sensitivity and effectivity of immune system against viral infections. Currently, antiviral therapy is available only for a limited number of zoonotic viral infections. Because viruses are intracellular parasites, antiviral drugs are not able to deactivate or destroy the virus but can reduce the viral load by inhibiting replication and facilitating the host's innate immune mechanisms to neutralize the virus.

Conclusion: Lessons from recent viral epidemics - Considering that certain nutraceuticals have demonstrated antiviral effects in both clinical and animal studies, further studies are required to establish their therapeutic efficacy. The components of nutraceuticals such as luteolin, apigenin, quercetin and chlorogenic acid may be useful for developing a combo-therapy. The use of probiotics to enhance immunity and immune response against viral infections is a novel possibility. The available antiviral therapy is inefficient in deactivating or destroying the infecting viruses, may help in reducing the viral load by inhibiting replication. The novel efficient antiviral agents are being explored.
More Information

*Address for Correspondence: Dr. Vinod Nikhra, MD, Hindu Rao Hospital \& NDMC Medical College, New Delhi, India, Tel: +919810874937;

Email: drvinodnikhra@gmail.com;

drvinodnikhra@rediffmail.com

Submitted: 07 March 2020

Approved: 08 April 2020

Published: 09 April 2020

How to cite this article: Nikhra V. The Transzoonotic Virome interface: Measures to balance, control and treat epidemics. Ann Biomed Sci Eng. 2020; 4: 020-027.

DOI: 10.29328/journal.abse.1001009

\section{ORCiD: orcid.org/0000-0003-0859-5232}

Copyright: @ 2020 Nikhra V. This is an open access article distributed under the Creative Commons Attribution License, which permits unrestricted use, distribution, and reproduction in any medium, provided the original work is properly cited.

Keywords: Virome interface; Zoonotic viral transmission; Viral epidemics; COVID-19; MERS; SARS; Nutraceuticals; Probiotics; Anti-viral agents

(A) Check for updates

(3) OPEn Access 


\section{The global viral communities}

The viruses are the most abundant biological entity on Earth. They have a global distribution, bear phylogenetic diversity and host specificity, and afflict bacteria, plants, animals and human population [1]. The term Virome refers to the viral metagenomes, which make up all the viral community associated with a particular ecosystem and includes both RNA and DNA viruses [2]. Taken together, the overall virome amounts to about $10^{31}$ viral particles on Earth. Further, the global virome is widespread, diverse and most of it remains uncharacterized, though broadly classified as plant, zoonotic and human viruses (Figure 1).

In general, characterizing viral communities is more challenging than bacteria, archaea and eukaryotes because viruses do not possess phylogenetically conserved genes. They are obligate intracellular parasites possessing single - or double-stranded DNA or RNA genomes. Viral infection begins when the viral surface proteins bind to receptor proteins on the host cell surface, followed by internalisation, replication and lysis. Alternatively, some viruses can remain dormant inside the host cells until conditions are favourable for their replication. While protein-protein interactions between virion and host are species specific in general, some viruses can have a broader host range.

The plant virome is composed of viral nucleic acids, DNA or RNA, and associated with a plant or community of plants. Plant viruses are harmful to the cultivated crop plants negatively affecting host morphology and physiology resulting in disease. Native wild/non-cultivated plants are often latently infected with viruses without any clear symptoms but pose a threat to cultivated crops because they can be transmitted by contact or vectors and cause disease.

The great majority of plant viruses have an RNA genome, which is usually small and single stranded (ss), but some viruses have double-stranded (ds) RNA, ssDNA or dsDNA genomes. Most plant viruses lack an envelope. The plant viruses can spread by direct transfer of sap by contact of a

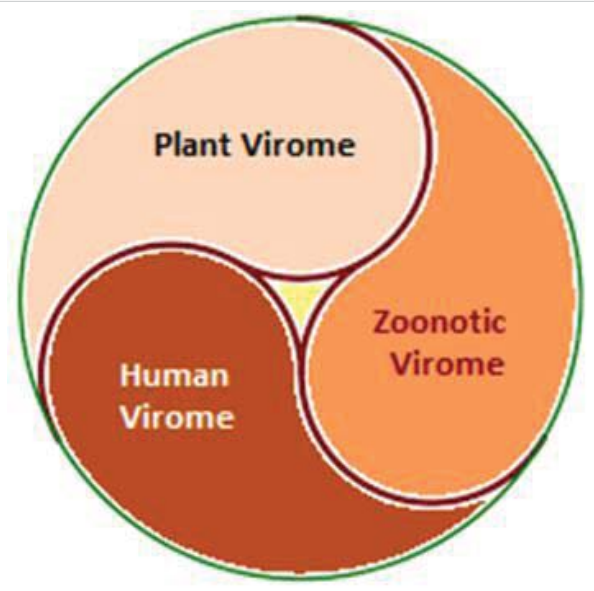

Figure 1: Global virome - Plant, Zoonotic and Human Viruses. wounded plant with a healthy one or transmitted by a vector, most often insects. Soil-borne nematodes also have been shown to transmit viruses by feeding on infected roots. Apart from this, a number of virus genera are transmitted, through soil borne zoosporic protozoa associated with the plant roots. In addition, plant virus transmission through generations may occur by seeds.

The human microbiome is comprised of communities of commensal, symbiotic and pathogenic bacteria, viruses, archaea, and small eukaryotes that actively interact with each other and the host to maintain homeostasis. The human virome is an essential part of the human microbiome and comprises of endogenous retroviruses, eukaryotic viruses and bacteriophages. The gut microbial genes are involved in nutrient synthesis, the metabolism of amino acids, carbohydrates and lipids and evolution and maintenance of immune system. The human virome is associated with Type-1 diabetes (T1D), Type-2 diabetes (T2D), Inflammatory Bowel Disease (IBD), Human Immunodeficiency Virus (HIV) infection and malignancy. Further, the trans-species interactions of viruses with bacteria, small eukaryotes and host are associated with various zoonotic viral diseases and disease progression.

\section{VIROME interface and transmission}

Plant viruses affect plants, especially larger plants. Like all other viruses, plant viruses are obligate intracellular parasites. The direct plant-to-human transmission is rare but has been found. There is evidence to suggest that the virus common to peppers, the Pepper Mild Mottle Virus (PMMoV), a member of Tobamo virus family, may infect humans and cause clinical symptoms [3]. The studies indicate that tobamoviruses are highly stable outside living host-cell. Another plant virus, Groundnut bud necrosis virus, one of the commonly occurring to spo virus in India, may have potential for host-switching to human or other animals.

The plant-based food and water are obvious route through plant viruses can get access to human body. The other possible route of access of plant virus directly to human cells is through insects that feed on both plant and human. These findings trigger to re-evaluate the dogmatic concept that plant viruses are safe to human health even though numerous viruses are consumed through various types of fresh foods and foodproducts [4].

Flora and fauna, plants and wildlife, especially mammals and birds, are hosts to an enormous number of viruses, which circulate in their specific echo-biospheres. These viruses usually inhabit asymptomatically in their natural hosts and occasionally, spill-over from their reservoir hosts to infect other species and cause disease states (Figure 2). The similar cross-species transmission from their natural reservoir hosts to infect human is also a rare probability leading to the zoonotic human viral infection, which is usually due to 


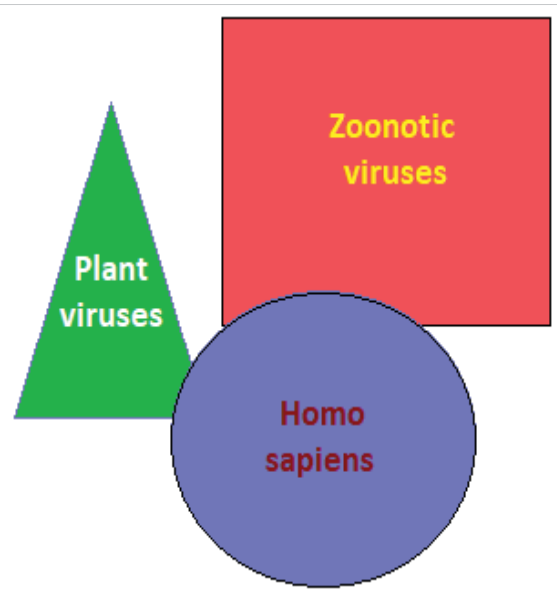

Figure 2: Trans-viral Interface and Spill-over

the viruses that spread from non-human animals (usually mammalian or avian hosts) to humans [5].

There are about 260 viruses known in humans and a multitude of unknown viruses represent potential cause of zoonoses [6]. Further, it is held that of about 1.6 million mammalian and avian viruses, only about 25 viral families may have potential to cause infections in human [7]. There is, both, an increased recognition of the emergence of zoonotic infections as well as the increased incidence of emerging zoonotic infections in modern times, though such an event may go undetected if there are no significant clinical symptoms or occurs at a small scale [8]. Still, these viruses remain undetected until they cause disease states in humans. Also, some unknown viral agents emerging from the wildlife reservoir causing limited disease outbreak in other animals, including humans, may go unrecognised. The better diagnostic methods and advanced in-depth investigations have led to the prompt recognition and epidemiological action. For example, the Hendra virus (HeV) disease in Australia in 1994 or Nipah virus (NiV) in Southern India in 2019 might have not been identified because of the small scale and random clusters.

The high pathogenicity of the emerging zoonotic viruses and their transmissibility in humans and non-reservoir species is important factor as compared to various indolent viral infections occurring and circulating in the human population. Still, the interspecies transmission of zoonotic agents from their natural reservoir host and its human-tohuman transmission is an uncommon event. The increased spill-over events lead to the increased probability of the emergence of a highly plastic and adapted virus capable of trans-human infections. Various factors like increased human settlements and encroachments, expanded travel and trade networks, altered wildlife and livestock practices, modernised and mass-farming practices, compromised ecosystems and habitat destruction, and global climate change are recognised to have impact on the interactions between virome and its hosts and other species and act as drivers of trans-species viral spill-over and human transmission.

\section{Zoonotic viral diseases and epidemics}

Zoonotic viral infections and epidemics: The zoonotic viruses have caused various deadly pandemics in human history. The Spanish flu in 1918-19 infected almost onethird of the global human population and caused 50-100 million deaths. More recently, Nipah, Hendra, Hanta and Ebola viruses, several influenza subtypes, and the SARS (severe acute respiratory syndrome) and MERS (Middle East respiratory syndrome) coronaviruses have caused limited albeit fatal outbreaks.

The infectious diseases are responsible for about one in five human deaths worldwide. In addition, they impose a heavy societal and economic burden on individuals, families, communities and countries [9]. Jones, et al. describing the emergence of 335 infectious diseases in the global human population between 1940 and 2004, concluded that nearly two-thirds the diseases originated in wildlife and the current global trends indicate that novel viral threats may continue to emerge at an accelerating rate [10]. These can be further characterized as either newly emerging or re-emerging infectious diseases, caused by pathogens that have infected the same host species earlier, but continue to appear in new locations or in drug-resistant forms, or reappear after their apparent control or elimination [11]. The predominance of zoonoses among the prevalent infectious diseases, underlines importance of the animal-human-ecosystem interface in disease transmission.

Further, the emergence of novel zoonotic pathogens is a challenge to global healthcare. Though, the advent of sophisticated diagnostics tools has improved our capacity for early detection, the exposure prophylaxis and post-exposure treatment modalities are insufficient. The emerging zoonoses leading to viral epidemics are potential health threats of modern times. Apart from various biological factors, the ecological, economic and developmental activities and behavioral practices influence the transmission interface, leading to dissemination and transmission through the human population. In addition, there are complex and dynamic factors such as human population density, mobility, lifestyle, behaviours and food choices linked to the zoonotic viral spillover, transmission and spread (Figure 3).

\section{Human coronavirus disease: COVID-19}

The Interspecies Transmission of Coronaviruses: There are distinct mechanisms which enable the interspecies transmission of coronaviruses. The coronavirus spike (S) glycoproteins can bind to analogous receptor proteins (receptor orthologs) in species other than their primary host [12]. Further, these viruses are capable of replicating in multiple hosts or a cluster of species. Thus, the SARSlike coronaviruses are capable of binding to angiotensin converting enzyme 2 (ACE2) receptors from multiple species, including humans [13]. Once they are able to infect 


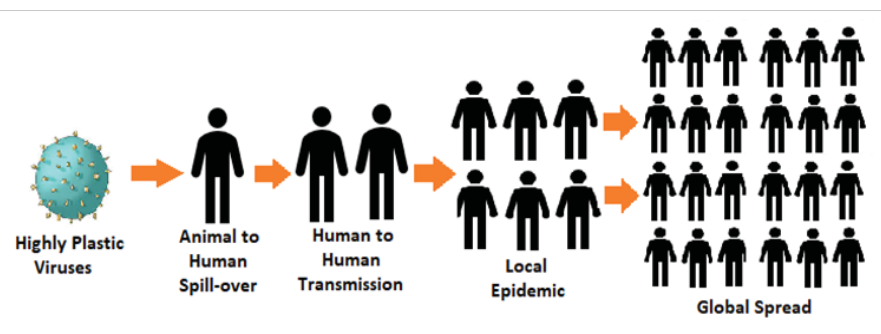

Figure 3: Epidemiology of Transmission and Global Spread of Zoonotic Viral Infections.

across species, mutations may arise permitting human-tohuman transmission [14]. The coronaviruses with a broader host range possess mutations in the $\mathrm{S}$ glycoprotein gene, a phenomenon rendering it capable of binding to a variety of host cell proteins or mutate further during docking and entry to host cells. The coronaviruses recognise various receptorproteins, including aminopeptidase N, ACE2, and dipeptidyl peptidase 4 (DPP4). Finally, some coronaviruses can use sugar moieties as receptors or co-receptors for entry, availing an alternative strategy for trans-species spill-over and transmission [15].

In addition, the coronavirus structure has some specific features. It has the S-glycoprotein embedded in the lipid bilayer surrounding the nucleocapsid, which mediates viral binding to host receptors. The S glycoproteins of SARS-CoV bind to ACE2 on the surface of host cells for docking and entry, whereas S-glycoproteins of MERS-CoV can bond to DPP4. The small and medium sized Chinese horseshoe bats belonging to Rhinolophidae family are natural reservoirs of SARS-CoV. As the studies have indicated, the intermediate hosts may not be necessary for direct human to human transmission for some SARS-like coronaviruses. The host age has also been identified as a factor in the cross-species transmission of coronaviruses. Further, in the infected persons, the age may influence the disease pathogenesis, clinical course as well as the prognosis.

The Pathogenicity of Coronaviruses: The coronaviruses have certain pathological features which promote their disease potential. Following SARS infection for the first 12 to 24 hours, there is no measurable interferon-stimulated genes (ISG) response in the infected airway epithelial cells. Later, some ISGs are activated at 24 hours, and most may follow and reach peak titres between 24 and 30 hours. Thus, by the time the cell-intrinsic defence mechanism gets turned on, the viruses have irreversibly damaged the cellular appendages [16]. The MERS infection carries a similar sequence of events. The significant delay in cell intrinsic immune recognition and ISG induction in SARS, MERS, to some extent in H5N1 and likely in COVID-19, downregulates a subset of the ISGs leading to immune response failure. The mechanism underlying this phenomenon appears to be epigenetically regulated and allows the viruses to manipulate host cell intrinsic response and increases the disease severity.

Over the past few months, COVID-19, a novel RNA coronavirus outbreak has infected more than 90,000 people and caused over 3,000 deaths. The lethality of COVID-19 is about 2.92 percent or more in some instances, being about 30 times or more lethal than the typical influenza. Both influenza and coronavirus (SARS, MERS and COVID-19) cause severe inflammation in the lungs, leading to viral pneumonia manifesting as acute respiratory distress, multi-organ failure and death.

\section{Measures to deal with epidemics like covid-19}

Nutraceuticals and other dietary constituents: It appears that certain nutraceuticals may provide relief in clinical symptoms to patients infected with encapsulated RNA viruses such as influenza and coronavirus (Figure 4). These nutraceuticals appear to reduce the inflammation in the lungs and help to boost type 1 interferon response to these viral infections [17]. The nutraceuticals that might help in controlling of RNA viruses including influenza and coronavirus include Ferulic acid, Lipoic acid, Spirulina, N-Acetylcysteine, Selenium Glucosamine, Zinc, Yeast BetaGlucan and Elderberry. They have been found to reduce the duration and severity of infection and reduce mortality in experimental animals infected with influenza [18].

Some recent discoveries have pointed the way to effective use of nutraceuticals for potentiating the type 1 interferon response to RNA viruses. The single-stranded viral RNA trapped within endosomes evokes superoxide production by NOX2-dependent NADPH oxidase complexes, which leads to an oxidation of Cys98 on toll-like receptor 7 (TLR7), blocking its ability to transmit a signal to stimulate type 1 interferon production [19]. These findings point to the possibility that nutraceuticals capable of inhibiting NOX2, promoting clearance of hydrogen peroxide or helping to restore of the native structure of Cys98 in TLR7, may be able to boost the TLR7-mediated induction of type 1 interferon and antiviral antibodies.

Further, it is known that heme oxygenase-1 (HO-1) induction potentiates the type 1 interferon response to influenza virus. Thus, the Phase 2 -inductive nutraceuticals - such as ferulic acid, lipoic acid and sulforaphane - which promote induction of HO-1, may be helpful in stimulating the type 1 interferon response. The downstream consequences

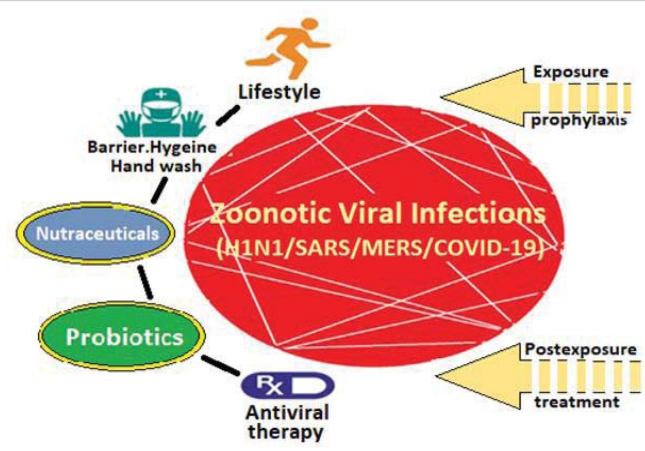

Figure 4: Prophylaxis and Treatment Modalities for Viral Infections Like COVID-19. 
of hydrogen peroxide may also be mitigated by phase 2-inductive nutraceuticals, which induce peroxidase enzymes and promote the synthesis of glutathione, a cofactor for certain peroxidases and a catalyst in reactions that reconvert oxidized cysteine groups to their native form. Selenium being a cofactor for certain peroxidases, ensuring adequacy of selenium nutrition might also be helpful. The selenium deficiency also increases the rate at which viruses can mutate, promoting the evolution of strains that are more pathogenic and capable of evading immune response. Zinc, also, supports function and proliferation of various immune cells.

The phycocyanobilin (PCB) chromophore of cyanobacteria (such as spirulina) and certain types of blue-green algae has been shown to possess NAPDH oxidase inhibiting activity and significant antioxidant and anti-inflammatory effects. Hence, the ingestion of spirulina or spirulina extracts enriched in PCB may potentiate the type 1 interferon response in the context of RNA virus infection. In animal experiments, oral administration of spirulina extract rich in phycocyanin has been found to decrease mortality in influenza-infected mice. The antioxidants can also protect lung parenchyma by quelling excessive inflammatory reaction. They can also decrease the inflammatory response, in general, both by suppressing viral spread and by reducing pro-inflammatory signaling in endothelial cells. The nutraceuticals like Black Currant, Jamaican Sorrel, bee pollen, Echinacea Purpurea, Siberian Ginseng, honey, bee propolis, and Goldenseal, and components of these nutraceuticals such as luteolin, apigenin, quercetin and chlorogenic acid may hold promise.

Glucosamine administration may up-regulate the mitochondrial antiviral-signaling (MAVS) protein activation, which is a key mediator for type 1 interferon response, leading to activation of cytosolic RNA virus detectors RIG-1 and MDA5, and subsequently, the activation of the transcription factor interferon regulatory factor 3 (IRF3). The mice fed on a glucosamine-enriched diet markedly enhances the survival of wild-type mice infected with influenza virus. The highdose glucosamine supplementation might aid prevention and control of RNA virus infections. The yeast beta-glucan also has immunostimulant effects by amplifying the dendritic cell activation via dectin-1 and CR3 receptors. It has been shown to enhance immune response in mice challenged with influenza virus [20]. Certain herbal preparations such as extracts of elderberry, a rich source of anthocyanins and their metabolite, ferulic acid, have shown potential for improving the symptoms of infections with influenza and other RNA viruses [21].

\section{Gut microbiota and probiotic therapy}

Human Gut Biosphere and Immunity: The gastrointestinal tract, skin and genitals, and various other body organs such as upper and lower respiratory tract and lungs, harbour large and diverse communities of bacteria, viruses and other microscopic life. The microbial ecosystems throughout the body interact with the molecular processes, which have been linked to various aspects of human physiology including immunity, and a disturbed microbiota is associated with deranged immune response (Figure 5).

The gut epithelium actively senses microbes, playing an essential role in maintaining host-microbial homeostasis at the mucosal interface. There has been documented a causal relationship between altered microbial communities, i.e. dysbiosis, immune response and disease. There is a crosstalk between the gut microbiota and host immune system. The human intestinal microbiota acting in tandem with the host's defence and immune system, is vital for preservation of health and the integrity and the activity of the gut microbes are responsible for the protection from disease states including viral infections. Certain metabolites or antigens presented by members of the microbiome may help raise the immune system's sensitivity and effectivity to viral infections. Further, the gut microbes harbour enzymes and secrete molecules that can influence drug absorption, metabolism, efficacy and toxicity.

\section{Gut Microbiome and viral infections}

During the in course of infection process, various viruses encounter the commensal microbiota of the hosts. It is possible that there are robust interactions between these viruses and the commensal microbiota. Thus, in the regulation of viral infection, commensal microbiota appears to play a variable but critical role. It may promote viral infectivity through diverse mechanisms but can also exert substantial inhibitory effects on viral infection. In addition to fostering the generation of immunoregulatory Treg cells, the commensal microbiota has antiviral effect by suppressing the activation of effector immune cells and by inhibiting the production of various inflammatory cytokines that are pivotal for virus elimination. On the downside, the commensal microbiota may facilitate genetic recombination of viruses and enhance their infectivity.

There is evidence of modulation of virus infectivity by the commensal microbiota of the host. During the infection process, viruses may have substantial and intimate interactions with the commensal microbiota. There is evidence that the commensal microbiota regulates and is in turn regulated by invading viruses through diverse mechanisms, thereby having stimulatory or suppressive roles in viral infections. Further,

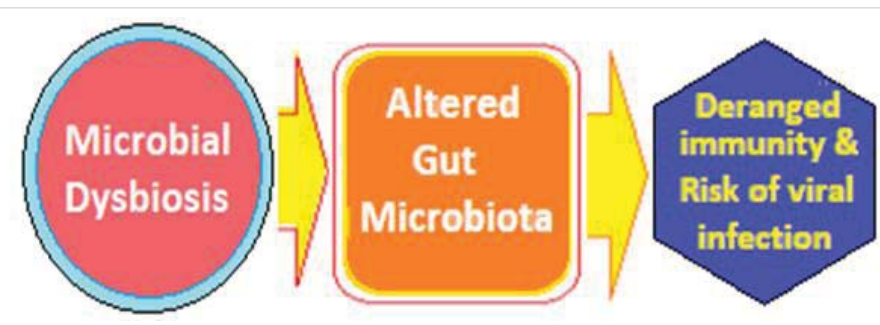

Figure 5: The Basis of Probiotic Treatment in Viral Infections. 
the commensal microbiota may modulate the efficiency of viral replication, transmission and persistence, and the outcome of the viral infection. The integrity of the commensal microbiota can be disturbed by invading viruses, causing dysbiosis in the host and further influencing virus infectivity [22]. On the other hand, the microbial dysbiosis may interfere with the absorption, metabolism and therapeutic efficacy of the antiviral and other supportive drugs given for treatment.

The Evidence from Experimental Studies: There is a potential for microbe-based probiotic adjunctive therapeutic approach to critical respiratory viral infections [23]. In general, the use of probiotics is related to the process through which the viruses activate the recognition and bonding of receptors and domains on the host cells and direct the replication of virions and alter the host immune response. Various animal and in vitro studies have identified of mechanisms underlying the immunomodulatory capacity of specific probiotics, which appears to be strain-specific and results from a combination of signaling pathways activated as a result of a specific microbe-derived ligands interacting with the corresponding pattern recognition receptors and domains on host cells. The probiotics induce changes in dendritic cell phenotype and function, T- cells, natural killer cells and alveolar macrophages forming the basis of the protective effect of probiotics.

The beneficial probiotic bacteria are demonstrated to promote the host defence and to modulate immune response in various viral infections [24]. The probiotics frequently include Lactobacillus or Bifidobacterium species, apart from other bacteria, including non-pathogenic forms of Escherichia coli and Bacteroides, as well as certain yeasts, such as Saccharomyces. The lactic acid bacteria (LAB) has shown functional antibacterial and antiviral activity against diverse human and animal viruses [25]. In the highly contagious, coronavirus induced transmissible gastroenteritis (TGE) causing severe diarrhoea and other symptoms leading to death in young piglets, the probiotic Lactobacillus plantarum strain N4(Lp) has been demonstrated to have protective effects. The in vitro Lp supplementation led to dose-dependent rescue of viability of infected cells and pre-treatment of cells with probiotic metabolic products reduced viral proliferation [26]. Another Lactobacillus strain, Lactobacillus rhamnosus GG protects mice from H1N1 influenza virus infection by regulating respiratory immune responses [27]. In a recent study, Lactococcus lactis strain Plasma (LC-Plasma) was shown to possess strong stimulatory activity for plasma cytoid dendritic cells via the TLR9-pathway to promote viral replication control [28].

Apart from the probiotic bacteria, their components are also able to induce potentially beneficial effects for host cells. The compounds, such as lactic acid, acetic acid and $\gamma$-aminobutyricacid produced by probiotic bacteria are capable of enhancing body immunity and controlling sepsis. The exopolysaccharides (EPSs) are biological high-molecular long- chain polysaccharides that are secreted by microorganisms. The EPS secreted by Lactobacillus acidophilus were found to inhibit TGE viral infection and improved levels of IFN-r, IL-6, IL-8 $[29,30]$. Further, the probiotic therapy has been shown to facilitate CD4 recovery in HIV-1 infected patients [31].

\section{Specific antiviral therapy}

The aim of antiviral therapy is to minimize symptoms and infectivity as well as to shorten the duration of illness. Currently, antiviral therapy is available only for a limited number of infections [32]. Because viruses are obligate, intracellular parasites, it is difficult to find drug targets that interfere with viral replication without harming the host cells. Unlike other antimicrobials, antiviral drugs do not deactivate or destroy the virus but aim reduce the viral load by inhibiting replication and facilitating the host's innate immune mechanisms to neutralize the virus. The most dreaded complication of an uncontrolled virus infection is sepsis, which is often underdiagnosed and has downhill course [33]. The virulent viruses causing viral sepsis, on one hand display the capacity to evade the immune system, whereas on the other, induce powerful inflammatory responses, often characterised by high levels of TNF- $\alpha$ and IL- 6 expression along with low IFN- $\gamma$ expression that can damage the host tissues.

The influenza viruses cause upper-respiratory infections in their hosts with symptoms such as severe lassitude, headache, chills, muscle aches and delayed mild cough with signs of fever. These systemic symptoms are due to the release of cytokines by the bronchial epithelial and macrophage cells. There are drugs which can inhibit influenza virus from entering or being effective inside the bronchial epithelial cells. The most commonly used drug for H1N1 is a neuraminidase inhibitor, oseltamivir, which prevents newly formed viruses from finalizing their budding from an infected cell.

Amantadine is an M2 ion channel blocker, whereas rimantadine is a weak NMDA receptor antagonist, Oseltamivir, zanamivir and peramivir are potent neuraminidase inhibitor and are recommended for both influenza treatment as well as prophylaxis. Baloxavir marboxil is a new influenza antiviral drug, approved recently. Baloxavir targets the cap-dependent endonuclease activity of influenza virus [34].

\section{Conclusion}

\section{Lessons from viral epidemics}

Considering that certain nutraceuticals have demonstrated antiviral effects in both clinical and animal studies, further studies are required to establish their therapeutic efficacy in the encapsulated RNA viral infections like H1N1, SARS, MERS and COVID-19, where definitive treatment modalities are being envisioned and explored.

Certain nutraceuticals (and herbs) decrease the cytokines levels to reduce inflammation and tissue injury. 
The components of various nutraceuticals such as luteolin, apigenin, quercetin and chlorogenic acid may be useful for developing a combo-therapy. By combining these compounds with other drugs showing efficacy against viral infection, more effective drug combinations may be made to treat the potentially deadly viral diseases.

Understanding how the commensal microbiota enhances viral infection, especially the molecular requirements for the microbiota-mediated promotion of viral infections, may lead to the development of novel, feasible antiviral strategies. The probiotic therapy is expected to be a rational adjunctive therapeutic modality for options for critical viral respiratory disease. The use of probiotics to enhance immunity and immune response against viral infections is a novel possibility. Apart from probiotic bacteria, their components can also induce potentially beneficial effects for host cells. The compounds, such as lactic acid, acetic acid and $\gamma$-aminobutyric acid, and EPSs produced by probiotic bacteria are capable of improving immune response body, limiting cytokine storms and regulating septicaemia. The available antiviral therapy is inefficient in deactivating or destroying the infecting viruses, though it may help in reducing the viral load by suppressing replication. The novel efficient antiviral agents are being explored.

\section{References}

1. Paez-Espino D, Eloe-Fadrosh EA, Pavlopoulos GA, Thomas AD, Huntemann M, et al. Uncovering Earth's virome. Nature. 2016; 536 425-430.

PubMed: https://www.ncbi.nlm.nih.gov/pubmed/27533034

2. Edwards RA, Rohwer F. Viral metagenomics. Nature Rev Microbiol. 2005; 3: 504-510.

PubMed: https://www.ncbi.nlm.nih.gov/pubmed/15886693

3. Colson P, Richet H, Desnues C, Balique F, Moal V, et al. Pepper Mild Mottle Virus, a Plant Virus Associated with Specific Immune Responses, Fever, Abdominal Pains, and Pruritus in Humans. PLoS ONE. 2010; 5: e10041.

PubMed: https://www.ncbi.nlm.nih.gov/pubmed/20386604

4. Zhang $T$, Breitbart $M$, Lee $W H$, Run JQ, Wei CL, et al. RNA viral community in human faeces: prevalence of plant pathogenic viruses. PLoS Biol. 2006; 4: e3.

PubMed: https://www.ncbi.nlm.nih.gov/pubmed/16336043

5. Mackenzie JS, Jeggo M. Reservoirs and vectors of emerging viruses. Curr Opin Virol. 2013; 3: 170-179.

PubMed: https://www.ncbi.nlm.nih.gov/pubmed/23491947

6. Carrol D, Watson B, Togami E, Daszak P, Mazet JA, et al. Building a global atlas of zoonotic viruses. Bull World Health. 2018; 96: 292-294. PubMed: https://www.ncbi.nlm.nih.gov/pubmed/29695886

7. Olival KJ, Hosseini PR, Zambrana-Torrelio C, Ross N, Bogich TL, et al Host and viral traits predict zoonotic spill-over from mammals. Nature. 2017; 546: 646-650.

PubMed: https://www.ncbi.nlm.nih.gov/pubmed/28636590

8. Morse SS, Mazet JA, Woolhouse M, Parrish CR, Carroll D, et al Prediction and prevention of the next pandemic zoonosis. Lancet. 2012; 380: 1956-1965.

PubMed: https://www.ncbi.nlm.nih.gov/pubmed/23200504
9. Lozano R, Naghavi M, Foreman K, Lim S, Shibuya K, et al. Global and regional mortality from 235 causes of death for 20 age groups in 1990 and 2010: A systematic analysis for the Global Burden of Disease Study 2010. Lancet. 2012; 380: 2095-2128.

PubMed: https://www.ncbi.nlm.nih.gov/pubmed/23245604

10. Jones KE, Patel NG, Levy MA, Storeygard A, Balk D, et al. Global trends in emerging infectious diseases. Nature. 2008; 451: 990-993. PubMed: https://www.ncbi.nlm.nih.gov/pubmed/18288193

11. Fauci AS, Morens DM. The perpetual challenge of infectious diseases. N Engl J Med. 2012; 366: 454-461.

PubMed: https://www.ncbi.nlm.nih.gov/pubmed/22296079

12. Bolles M, Donaldson E, Baric R. SARS-CoV and emergent coronaviruses: Viral determinants of interspecies transmission. Curr Opin Virol. 2011; 1: 624-634.

PubMed: https://www.ncbi.nlm.nih.gov/pubmed/22180768

13. Graham RL, Baric RS. Recombination, reservoirs, and the modular spike: Mechanisms of coronavirus cross-species transmission. J Virol. 2010; 84: 3134-3146.

PubMed: https://www.ncbi.nlm.nih.gov/pubmed/19906932

14. Graham RL, Donaldson EF, Baric RS. A decade after SARS: Strategies for controlling emerging coronaviruses. Nature Rev Microbiol. 2013; 11: 836-848.

PubMed: https://www.ncbi.nlm.nih.gov/pubmed/24217413

15. Li F. Receptor recognition and cross-species infections of SARS coronavirus. Antiviral Res. 2013; 100: 246-254.

PubMed: https://www.ncbi.nlm.nih.gov/pubmed/23994189

16. Johnson CK, Hitchens PL, Evans TS, Goldstein T, Thomas K, et al. Spillover and pandemic properties of zoonotic viruses with high host plasticity. Sci Rep. 2015; 5: 14830.

PubMed: https://www.ncbi.nlm.nih.gov/pubmed/26445169

17. McCarty MF, DiNicolantonio JJ. Nutraceuticals have potential for boosting the type 1 interferon response to RNA viruses including influenza and coronavirus. Prog Cardiovasc Dis. 2020.

PubMed: https://www.ncbi.nlm.nih.gov/pubmed/32061635

18. Brayden Humpherys B, Busath DD. Anti-Influenza Nutraceuticals: Antiviral and Anti-Inflammatory Effects. Advances in Complementary \& Alternative medicine. 2019; 4.

19. To EE, Luong R, Diao J, O' Leary JJ, Brooks DA, et al. Novel endosomal NOX2 oxidase inhibitor ameliorates pandemic influenza $A$ virusinduced lung inflammation in mice. Respirology. 2019; 24: 1011-1017. PubMed: https://www.ncbi.nlm.nih.gov/pubmed/30884042

20. Vetvicka V, Vetvickova J. Glucan supplementation enhances the immune response against an influenza challenge in mice. Ann Transl Med. 2015; 3: 22.

PubMed: https://www.ncbi.nlm.nih.gov/pubmed/25738142

21. Hawkins J, Baker C, Cherry L, Dunne E. Black elderberry (Sambucus nigra) supplementation effectively treats upper respiratory symptoms: a meta-analysis of randomized, controlled clinical trials. Complement Ther Med. 2019; 42; 361-365.

PubMed: https://www.ncbi.nlm.nih.gov/pubmed/30670267

22. Li N, Ma W-T, Pang M, Fan QL, Hua JL. The Commensal Microbiota and Viral Infection: A Comprehensive Review. Front Immunol. 2019; 10: 1551.

PubMed: https://www.ncbi.nlm.nih.gov/pubmed/31333675 .

23. Lehtoranta L, Pitkäranta $A$, Korpela R. Probiotics in respiratory virus infections. Eur J Clin Microbiol Infect Dis. 2014; 33: 1289-1302. PubMed: https://www.ncbi.nlm.nih.gov/pubmed/24638909

24. O'Toole PW, Marchesi JR, Hill C. Next-generation probiotics: the spectrum from probiotics to live biotherapeutics. Nat Microbiol. 2017; 2: 17057.

PubMed: https://www.ncbi.nlm.nih.gov/pubmed/28440276 
25. Maeda N, Nakamura R, Hirose Y, Murosaki S, Yamamoto Y, et al. Oral administration of heat-killed lactobacillus plantarum I-137 enhances protection against influenza virus infection by stimulation of type interferon production in mice. Int Immunopharmacol. 2009; 9; 11-22. PubMed: https://www.ncbi.nlm.nih.gov/pubmed/19410659

26. Yang $\mathrm{Y}$, Song $\mathrm{H}$, Wang L, Palissa C, Esch B, et al. Antiviral Effects of a Probiotic Metabolic Products against Transmissible Gastroenteritis Coronavirus. Arch Virol. 2013; 158: 799-807.

PubMed: https://www.ncbi.nlm.nih.gov/pubmed/23188495

27. Harata G, He F, Hiruta N, Kawase M, Kubota A, et al. Intranasal administration of Lactobacillus rhamnosus GG protects mice from H1N1 influenza virus infection by regulating respiratory immune responses. Lett Appl Microbiol, 2010; 50; 597-602.

PubMed: https://www.ncbi.nlm.nih.gov/pubmed/20438620

28. Horie A, Tomita Y, Ohshio K, Fujiwara D, Fujii T. Characterization of genomic DNA of lactic acid bacteria for activation of plasmacytoid dendritic cells. BMC Microbiol. 2019; 19: 88.

PubMed: https://www.ncbi.nlm.nih.gov/pubmed/31060586

29. Kumar R, Seo BJ, Mun MR, Kim CJ, Lee I, et al. Putative probiotic lactobacillus spp. from porcine gastrointestinal tract inhibit transmissible gastroenteritis coronavirus and enteric bacterial pathogens. Trop Anim
Health Prod. 2010; 42: 1855-1860.

PubMed: https://www.ncbi.nlm.nih.gov/pubmed/20623187

30. Chai W, Burwinkel M, Wang Z, Palissa C, Esch B, et al. Antiviral effects of a probiotic enterococcus faecium, strain against transmissible gastroenteritis coronavirus. ArchlVirol. 2013; 158: 799-807. PubMed: https://www.ncbi.nlm.nih.gov/pubmed/23188495

31. Lu W, Feng $\mathrm{Y}$, Jing $\mathrm{F}$, Han $\mathrm{Y}$, Lyu N, et al. Association between gut microbiota and CD4 recovery in HIV-1 infected patients. Front Microbiol. 2018; 9: 1451.

PubMed: https://www.ncbi.nlm.nih.gov/pubmed/30034377

32. Razonable RR. Antiviral Drugs for Viruses Other Than Human Immunodeficiency Virus. Mayo Clin Proc. 2011; 86: 1009-1026. PubMed: https://www.ncbi.nlm.nih.gov/pubmed/21964179

33. Lin GL, McGinley JP, Drysdale SB, Pollard AJ. Epidemiology and Immune Pathogenesis of Viral Sepsis. Front Immunol. 2018; 9: 2147. PubMed: https://www.ncbi.nlm.nih.gov/pubmed/30319615

34. Fukao K, Ando Y, Noshi T, Kitano M, Noda T, et al. Baloxavir marboxil, a novel cap-dependent endonuclease inhibitor potently suppresses influenza virus replication and represents therapeutic effects in both immunocompetent and immunocompromised mouse models. PLoS One. 2019;14: e0217307.

PubMed: https://www.ncbi.nlm.nih.gov/pubmed/31107922 